\title{
Tension Optimization for a Cable-Driven Parallel Robot with Non- Negligible Cable Mass
}

\author{
Peng $\mathrm{Liu}^{1,2, *}$ and Yuanying Qiu ${ }^{1}$ \\ ${ }^{1}$ Key Laboratory of Ministry of Education for Electronic Equipment Structure Design, Xidian University, Xi'an, 710071, \\ P.R. China \\ ${ }^{2}$ School of Mechanical Engineering, Xi'an University of Science and Technology, Xi'an 710054, P.R. China
}

\begin{abstract}
This paper addresses the cable tension distributions of a cable-driven parallel robot with non-negligible cable mass for large dimension mechanisms. A well-known model which describes the profile of a cable under the action of its own weight allows us to take the cable sags into account. In addition, an approach to computing the cable tensions is presented, in which there are two major steps to obtain the cable tensions. In more detail, the first one is an iterative process for obtaining the iterative cable tensions, while the other one is the optimization of the cable tensions obtained by the first step. Finally, a large scale cable-driven manipulator currently under development is analyzed. The results show that cable sags have a significant effect on the cable tensions of such manipulators.
\end{abstract}

Keywords: Cable-Driven Parallel Robot, Cable Sag, Tension Optimization.

\section{INTRODUCTION}

Cable-driven parallel robots, possessing several promising advantages over their rigid-link counterparts, are a special class of parallel manipulators in which the moving platform is driven by cables instead of rigid links [1-3]. Based on the number of cables $(\mathrm{m})$ and the number of degrees of freedom of the platform (n), cable-driven parallel robots are classified into three categories, i.e. the incompletely restrained positioning mechanisms $(m<n+1)$, the completely restrained positioning mechanisms $(m=n+1)$ and the redundantly restrained positioning mechanisms $(m>n+1)[2,4]$. In most studies of cable-driven parallel robots, the cables are considered to be straight lines, as long as cables are massless and its tensions remain positive. Thereby, studies become much easier and, in most cases, the model obtained is quite close to reality $[5,6]$. But, in some particular cases, more complex cable behaviors must be taken into account to fit well with the real manipulator. Another aspect that has to be taken into account is sags of the long-span cables. Cables with significant mass will tend to sag under their own weight $[1,3,7-13]$.

Determining the optimal cable tension distribution is essential for the efficient control and operation of the cabledriven parallel robots. A few researchers have worked on the determination of the cable tensions for the cable-driven parallel robots. Generally, the cables of a parallel cable-driven robot are considered to be massless. Fang et al. [14] developed an analytical method to optimize cable tension distributions in cable-driven parallel robots based on minimizing the

*Address correspondence to this author at the School of Mechanical and electronic Engineering, Xidian University, Xi'an, Shaanxi, 710071, P.R. China; Tel: +86 88202451; Fax: +86 88202451;

E-mail: 200304405liupeng@163.com sum of cable tensions at every pose. Their method, however, is not applicable to cable-driven parallel robots with more than one redundant cable. Gosselin et al. [15] presented a non-iterative algorithm of cable tension determination and proposed four optimal objective functions for the algorithm, and the algorithm of cable tensions is just applicable to the completely restrained cable-driven parallel robots. Liu et al. [16] investigated optimal cable tension distributions for the cable-based parallel robots, in which the minimum cable tension distributions in the workspace are discussed. These method and algorithm above, indeed, are rationale for the cable-driven parallel robots in which the cables can be modeled as the massless inextensible straight lines rather than cable catenary model. However, this assumption, for the cable-driven parallel robots with long-span cables, is not rational owning to the sags caused by the non-negligible self-mass. In addition, the effects of the cable sags on both the kinematics and the cable tensions must be taken into account for large-span cables [8-11]. Riehl et al. [1, 3] proposed a method to determine both the kinematics and the cable tensions of minimally constrained cable-driven manipulators. But the proposed method cannot be applied to other cable-driven parallel robots in which the number of cables $\mathrm{m}$ exceeds the number of degrees of freedom of the platform $n$. Indeed, all of the above literatures, to the best of our knowledge, do not deal with the cable tension distributions for the redundantly restrained cable-driven parallel robots with non-negligible cable mass.

This paper mainly focuses on the aspect of the cable sags. Based on a well-known model of a cable with non-negligible mass, described in section 2, the numerical computations of the cable tensions for the redundantly restrained cable-driven parallel robots are presented in section 3 , in which an iterative optimization algorithm to solve the cable tensions, for a given pose, is proposed. This paper studies the effects of the 


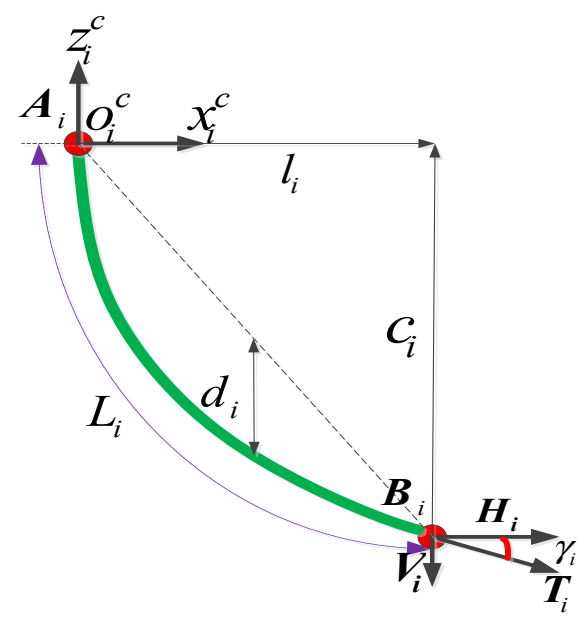

Fig. (1). Catenary cable in its vertical plane.

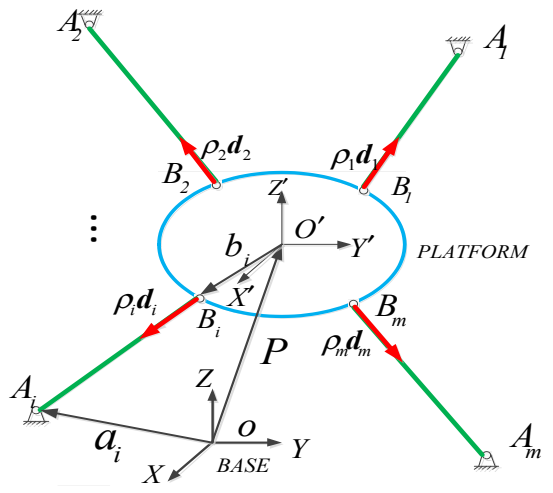

Fig. (2). Schematic of a cable-driven parallel robot.

cable sags on the cable tensions. And furthermore, the differences between the massless and the sags models regarding the determination of the cable tensions are discussed in section 4. Finally, section 5 concludes this paper.

\section{CABLE MODEL AND OPTIMAL CABLE TEN- SIONS}

In order to make the cable-driven parallel robots move stably and reliably, a uniform motion of the end-effector is a requirement. Indeed, in many situations, an inextensible catenary of non-negligible mass is likely to be an appropriate modeling of the cables of the robots with long-span cables [13]. As the result, the cables are modeled as inextensible catenary, while the inertias of the cables and platform can be ignored. It is well known that the profile of the cable catenary and the cable tensions are coupled. Contrary to the case of massless cable modeling, the cable tensions depend on the external forces acting on the cables but also on their own mass of the cables $[1,3]$. Cable structures have also complex mechanics behaviors because of their nonlinearity in a geometric sense. Thus, complete modeling for the cable-driven parallel robots with the catenary cables must be done.

\subsection{Cable Catenary Model}

The first challenge for analyzing a cable-driven robot with sagging cables is to mathematically describe the shape of the cables under the influence of gravity. One end of the homogeneous cable, in our model introduced here, is connected to the motor while the other end is connected to the end-effector. As shown in Fig. (1), a local cable frame, noted $\left\{o_{i}{ }^{\mathrm{c}} x_{i}{ }^{\mathrm{c}} z_{i}^{\mathrm{c}}\right\}$, is attached to $\boldsymbol{B}_{i}$ in Fig. (2), in which the $z^{\mathrm{c}}$ axis of the cable frame points in the same direction with the global $z$ axis. The non-elastic cables with significant mass will tend to sag under their own weights. Therefore, the inextensible catenary equation can be applied for describing the profile of the cables under their sags $[7,9]$. As a consequence, the profile of the cable $i$ is the catenary within the $o^{c} x^{c} z^{c}$ plane, which can be expressed as follows [9]:

$z_{i}^{c}=\frac{H_{i}}{\rho g}\left[\cosh \alpha_{i}-\cosh \left(\frac{2 \beta_{i} x_{i}^{c}}{l_{i}}-\alpha_{i}\right)\right], i=1,2, \mathrm{~L}, m$

where $x_{i}^{c}$ and $z_{i}^{c}$ are $x$ and $z$-direction coordinates in $\left\{o_{i}{ }^{\mathrm{c}} x_{i}{ }^{\mathrm{c}} z_{i}^{\mathrm{c}}\right\} ; \rho$ is linear density of the inextensible cable; $g=9.8 \mathrm{~m} / \mathrm{s}^{2}$ is the gravitational acceleration with the direction 
along the negative direction of the $z$ axis; $\alpha_{i}=\sinh ^{-1}\left[\frac{\beta_{i}\left(c_{i} / l_{i}\right)}{\sinh \beta_{i}}\right]+\beta_{i}, \beta_{i}=\frac{\rho g l_{i}}{2 H_{i}} ; H_{i}$ and $V_{i}$ are the horizontal and vertical components of each cable tension at the cable end $\boldsymbol{B}_{i} ; l_{i}$ and $c_{i}$ are the horizontal and vertical spans of each cable respectively.

It must be noted from Eq. (1) that this kinematics model with inextensible catenary cables differs from the traditional kinematics with the straight line cables for the cable-driven robots. As we can see from the number of the unknowns, we must take into account both the cable tensions and the cable lengths simultaneously because, in this case, we cannot separate both the aspects, the cable lengths depending directly on the cable tensions according to the cable sagging model described in Eq. (1). Furthermore, the slope of catenary at the last node $\boldsymbol{B}_{i}$ can be obtained from Eq. (1) as follows:

$\tan \gamma_{i}=\frac{\partial z_{i}^{c}}{\partial x_{i}^{c}}=-\sinh \left(\frac{2 \beta_{i} x_{i}^{c}}{l_{i}}-\alpha_{i}\right), i=1,2, \cdots, m$

where $\gamma_{i}$ is the angle between the tangent and the horizontal plane at the last node of the catenary.

It is noted from Eq. (2) that the slope of a catenary is relevant to the parameter $\beta_{i}$ being a function of the horizontal component $H_{i}$ of each cable tension. Thus the profiles of the cable catenaries, as mentioned before, are completely coupled to the forces applied to the cables, and therefore, these variables (cable lengths and cable tensions) have to be determined at the same time.

\subsection{Kinetostatic Model of a Cable-Driven Parallel Robot}

In order to make a cable-driven parallel robot completely controllable, it must be actuated redundantly due to the unilateral constraints nature of cables. The cable-driven parallel robot, as shown in Fig. (2), consists mainly of a moving platform connected to the fixed pulleys by the cables and the fixed base. Each pulley can be attached to the ceiling of a mast. As a result, the moving platform moves freely in every direction because the cables can be shortened and lengthened controlled by servo motors mounted to the fixed base.

When the cable sags are non-negligible, the cable tension distributions of cable-driven parallel robot become quite different from the ones with straight lines. The cable tension distributions must be solved as a problem solving the statics and the kinematics simultaneously rather than as a pure statics problem. In this section, the cable tension distributions for a cable-driven parallel robot are posed, and a solution method is presented. The cable tension distributions can be regarded as an iterative optimization problem. With regard to the completely restrained cable-driven parallel robots, the equations for this type of system consist of a set of statics equations and a set of kinematic-static equations. The position and orientation of the end-effector are specified, and therefore, the unknown variables are the cable lengths and the cable tensions. Thus, for a manipulator with $m$ cables, there will be a total of $2 m$ unknowns which are cable length and cable tension for each cable. And furthermore, there are $m$ kinematic-static equations which come directly from Eq. (1), while there are $n$ equations for static equilibrium, and they can be written as:

$$
\left\{\begin{array}{l}
\sum_{i=1}^{m} \boldsymbol{R}_{i}\left(-\boldsymbol{T}_{i}\right)+m g+\boldsymbol{F}_{e}=0 \\
\sum_{i=1}^{m} \boldsymbol{b}_{i} \times \boldsymbol{R}_{i} \boldsymbol{T}_{i}+\boldsymbol{M}_{e}=0
\end{array}\right.
$$

where $\boldsymbol{R}_{i}$ is the rotation matrix that maps the force vector $\boldsymbol{T}_{i}$ from the cable frame to the fixed/manipulator frame; $\boldsymbol{b}_{i}$ is the position vector of the end point of the $i$ th cable attached to the platform, and expressed in the frame attached to the center of gravity of the platform; and $g$ is the acceleration of gravity.

Combining with the Eqs. (1) and (2), Eq. (3) can be written in the following matrix form:

$\boldsymbol{J H}=\boldsymbol{W}$

where $\boldsymbol{J}$ is the structure matrix associated the driving cable; $\boldsymbol{H}=\left[H_{1}, H_{2}, \cdots, H_{\mathrm{m}}\right]^{\mathrm{T}}$ is the vector describing horizontal components of all the cable tensions at their last node; $\boldsymbol{W}=\boldsymbol{f}_{g}+\boldsymbol{W}_{\mathrm{e}}$, $\boldsymbol{f}_{g}=\left[\begin{array}{llllll}0 & 0 & m g & 0 & 0 & 0\end{array}\right]^{\mathrm{T}} \in \mathbf{R}^{6 \times 1}$ in which $m$ is the mass of the moving platform, and $\boldsymbol{W}_{\mathrm{e}}=\left[\boldsymbol{F}_{e} \boldsymbol{M}_{e}\right]^{\mathrm{T}} \in \mathbf{R}^{6 \times 1}$ is the external wrench.

It should be noted that the structure matrix $\boldsymbol{J}$ is relevant to the vector $\boldsymbol{H}$ through its dependency in $\tan \gamma_{i}$ being a function of the horizontal component $H_{i}$ of each cable tension. Thus, Eq. (4), formally, seems to be a linear equation but it is not substantially, since the relationship between the coefficient matrix $\boldsymbol{J}$ and the vector $\boldsymbol{H}$ is not straightforward. Actually, Eq. (4) is a non-linear equation in terms of the vector $\boldsymbol{H}$ describing horizontal components of all the cable tensions because the structure matrix $\boldsymbol{J}$ is relevant to the vector $\boldsymbol{H}$, in general, which is solved using an iterative algorithm employing the cable tensions and lengths obtained by massless straight line model as the initial iterative values here. And in more detail, the iterative algorithm can be shown as follows: Firstly, giving the end-effector platform position $\boldsymbol{X}_{k}$ in the workspace (the $k$ th platform position is denoted by the subscript $k$ ), the initial cable tensions, $\boldsymbol{H}^{(0)}$, can be obtained with the massless straight line model, while the slope of catenary at the first node, $\tan \gamma_{i}^{(0)}$, can be also obtained using Eq. (2). Secondly, substituting $\tan \gamma_{i}^{(0)}$ into the structure matrix $\boldsymbol{J}$ of the equation (4), the new vector $\boldsymbol{H}^{(1)}$ can be calculated using the equation (4). Then, substituting the new vector $\boldsymbol{H}^{(1)}$ into Eq. (2), the associated slope of cable catenary, $\tan \gamma_{i}^{(1)}$, can be obtained. And moreover, substituting $\tan \gamma_{i}^{(1)}$ into the equation (4), the new vector $\boldsymbol{H}^{(2)}$ can be calculated using the equation (4). Repeating the process until the vector $\boldsymbol{H}^{(j)}$ satisfies the convergence condition $\left(\left\|\boldsymbol{H}^{(j)}-\boldsymbol{H}^{(j-1)}\right\| \leq \alpha\right.$, $\alpha$ is the iterative stop threshold). The vector $\boldsymbol{H}^{(j)}$ is considered to be the solution to the horizontal components of the cable tensions.

There may be a group of the vectors satisfying the above convergence condition because the number of cables exceeds 
the degree of freedom of the platform for a completely restrained cable-driven parallel robot. Thus, an optimization algorithm is required to obtain a unique solution to the vector $\boldsymbol{H}$ which can confirm that the cable-driven parallel robot operation as stable as possible.

\section{OPTIMAL MODEL OF THE CABLE TENSION DISTRIBUTION}

\subsection{Cable Tension Equation}

Because the cable-driven parallel robots are redundant systems having more actuators than the task dimensions, infinitely many solutions for the distribution of cable tensions can be obtained for a given external load. One of the issues in the operation of the cable-driven parallel robots is resolving the actuation redundancy and determining the optimum cable forces distribution. Therefore, Eq. (4) from which the vector $\boldsymbol{H}$ can be obtained may have infinite solutions. In addition, the vector $\boldsymbol{H}^{(j)}$ satisfying the convergence condition is obtained with the iterative algorithm above, and furthermore, the structure matrix $\boldsymbol{J}$ is constant values substituting the vector $\boldsymbol{H}^{(j)}$ into the equation (4). And therefore, with the introduction of Moore-Penrose generalized inverse matrix $\left(\boldsymbol{J}\left(\boldsymbol{H}^{(j)}\right)\right)^{+}$of the structure matrix $\boldsymbol{J}\left(\boldsymbol{H}^{(j)}\right)$, the vector $\boldsymbol{H}$ can be expressed as follows $[2,9]$ :

$\boldsymbol{H}=\boldsymbol{H}_{s}+\boldsymbol{H}_{h}$

where $\boldsymbol{H}_{s}=\left(\boldsymbol{J}\left(\boldsymbol{H}^{(j)}\right)\right)^{+} \boldsymbol{W}$ is the special solution to the vector $\boldsymbol{H}$; $\boldsymbol{H}_{h}=\boldsymbol{N}\left(\boldsymbol{J}\left(\boldsymbol{H}^{(j)}\right) \lambda\right.$ is the homogeneous solution to the vector $\boldsymbol{H}$; $\boldsymbol{H}^{(j)}$ is the solution to the horizontal components of the cable tensions using the iterative algorithm; $\lambda$ is an arbitrary scalar.

The cable tension of cable $i$ at the cable node $\boldsymbol{B}_{i}$ is obtained from the following equation:

$T_{i}=H_{i} \sqrt{1+\tan ^{2} \gamma_{i}}(i=1,2, \mathrm{~L} m)$

In order to ensure the normal work of the cable-driven parallel robot, the tension vector $\boldsymbol{T}$ must satisfy the following condition:

$\boldsymbol{T}_{s, \min } \leq \boldsymbol{T} \leq \boldsymbol{T}_{s, \max }$

where $\boldsymbol{T}=\left[T_{1}, T_{2}, \cdots, T_{m}\right]^{\mathrm{T}} \in$ is the vector consisting of all cable tensions; the lower bound of the cable tension $\boldsymbol{T}_{s, \min }=\left[T_{1, \min }, T_{2, \min }, \cdots, T_{m, \text { min }}\right]^{\mathrm{T}}$ is required to keep cables taut; while upper bound of the cable tension $\boldsymbol{T}_{s, \max }=\left[T_{1, \max }\right.$, $\left.T_{2, \max }, \cdots, T_{m, \max }\right]^{\mathrm{T}}$ is limited by the output torques of the servo motors and the maximum tension the cable can withstand without breaking.

\subsection{Optimal Model of the Cable Tension Distribution}

It can be shown from Eq. (5) that the homogeneous solution $\boldsymbol{H}_{h}$ is infinite due to the arbitrariness of $\lambda$. Therefore, in order to obtain a unique solution, the minimum variance having the least differences among all cable tensions and the arithmetic mean value of them is used to optimize while using Eq. (5) and (7) as the constraint conditions. Mathematically, the determination of the vector $\boldsymbol{H}$ can be formulated as follows:

$$
\begin{array}{ll}
\text { Object } & \min \left(\frac{1}{m}\left[\sum_{i=1}^{m}\left(H_{i}-E(\boldsymbol{H})\right)^{2}\right]\right) \\
\text { subject to } & \boldsymbol{J}\left(\boldsymbol{H}^{(j)}\right) \boldsymbol{H}=\boldsymbol{Q}\left(\boldsymbol{H}^{(j)}\right) \\
& \boldsymbol{T}_{{ }_{\text {min }}^{s}} \leq \boldsymbol{T} \leq \boldsymbol{T}_{\text {max }}^{s}
\end{array}
$$

where $E(\boldsymbol{H})=\left(H_{1}+H_{2}+\cdots+H_{m}\right) / m$ is the arithmetic mean value of the vector $\boldsymbol{H}$.

As mentioned above, particular importance will be attached to the fact that Eq. (4) is a highly non-linear equation. In order to solve the nonlinear equation, we present an iterative optimization algorithm to solve the vector $\boldsymbol{H}$, as mentioned previously, which employs the cable tensions and lengths obtained by massless straight line model as the initial iterative values. Here, the iterative optimization algorithm can be summarized as follows:

1. Input the end-effector platform position $\boldsymbol{X}_{k}$ in the workspace and the cable density $\rho$, and obtain $l_{i}, c_{i}$ and $\theta_{i}\left(\theta_{i}\right.$ is the angle between $x^{\mathrm{c}}$ axis of $o^{\mathrm{c}} x^{\mathrm{c}} z^{\mathrm{c}}$ and $X$ axis of $O X Y Z$; the $i$ th cable is denoted by the subscript $i$ ); $k=1$ (the $k$ th end-effector platform position is denoted by the subscript $k$ ).

2. Calculate the initial value $\tan \gamma_{i}^{(0)}=c_{i} / l_{i}$, and obtain the initial value $\boldsymbol{J}^{(0)}=\left[J^{(0)}, J^{(0)}{ }_{2}, \cdots, J^{(0)}{ }_{m}\right]^{\mathrm{T}}$; and then, calculate the initial value of the vector $\boldsymbol{H}$ using Eq. (4), $\boldsymbol{H}^{(0)}=\left(\boldsymbol{J}^{(0)}\right)^{+} \boldsymbol{W} ; \boldsymbol{j}=1$ (the $j$ th iterative step vector $\boldsymbol{H}$ is denoted by the superscript $j$ ).

3. Obtain the associated slope of cable catenary $\tan \gamma_{i}^{(j)}$ by substituting the vector $\boldsymbol{H}^{(j-1)}$ into Eq. (2).

4. Substitute $\tan \gamma_{i}^{(j)}$ into the equation (4) and update the new structure matrix $\boldsymbol{J}^{(j)}$, and then calculate the new vector $\boldsymbol{H}^{(j)}$ using the equation (4), $\boldsymbol{H}^{(j)}=\left(\boldsymbol{J}^{(j)}\right)^{+} \boldsymbol{W}$.

5. Judge that the predetermined condition $\left\|\boldsymbol{H}^{(j+1)}-\boldsymbol{H}^{(j)}\right\| \leq \alpha(\alpha$ is the iterative stop threshold $)$ is satisfied or not. If it is, stop the iterative process and record the vector $\boldsymbol{H}^{(j)}$ satisfying the nonlinear equation (4). If not, go to the 4th step, and repeat the process from the steps (3)-(5), $j=j+1$.

6. Substitute the vector $\boldsymbol{H}^{(j)}$ satisfying the nonlinear equation (4) into the right of Eq. (5), obtain $\left(\boldsymbol{J}\left(\boldsymbol{H}^{(j)}\right)\right)^{+} \boldsymbol{W}+\boldsymbol{N}\left(\boldsymbol{J}\left(\boldsymbol{H}^{(j)}\right) \lambda\right.$, and then, calculate the optimized vector $\boldsymbol{H}_{o, k}$ (the optimized vector is denoted by the subscript $o$ ) according to the optimal model Eq. (8).

7. Calculate the cable tension of the $i$ th cable $T_{i}$ substituting the optimal vector $\boldsymbol{H}_{o, k}$ into Eq. (6).

8. Judge whether the camera platform position $\boldsymbol{X}_{k}$ is the last position. If it does not, go to step (1) and solve the cable tensions of the next position, $k=k+1$; If it does, record and output the optimized vector of $\boldsymbol{H}$ and the cable tension $T_{i}$ of the $i$ th cable, stop the calculation.

It is noted that, observing the iterative optimization algorithm above, the steps (1)-(5) are employed to obtain the 
solution of the cable tensions using an iterative method, in which the steps (1)-(2) are used to calculate the initial values for the iteration process. And moreover, the structure matrix $\boldsymbol{J}$ in Eq. (4) is determined by substituting the solution of the cable tensions using the iterative method into the left of Eq. (3), and therefore, Eq. (4) can be considered as a linear equation. The vector $\boldsymbol{H}$, in this case, can be optimized because Eq. (4) is an indeterminate equation having infinitely many solutions. While the steps (6)-(7) are used to obtain the optimized solution to the vector $\boldsymbol{H}$ at the present end-effector platform position. From above, it can be seen that there are two major steps to obtain the horizontal components of the cable tensions here. The first one is the step being used to solve the nonlinear equation (4) with an iterative algorithm employing the cable tensions and lengths obtained by massless straight line model as the initial iterative values, and therefore, the solution of the equation (4) can be obtained. And the other one is the optimization of the cable tension solutions obtained by the first step, and furthermore, the optimized solution of the cable tensions can be received. Generally speaking, the horizontal components of the cable tensions, $\boldsymbol{H}$, can be obtained with the iterative algorithm in the first step without the optimization of it. However, the optimization procedure with which the optimized vector $\boldsymbol{H}$ can be obtained, in our manuscript, is required to confirm that the cable-driven parallel robot operations as stable as possible. It should be pointed that the optimized vector $\boldsymbol{H}_{o, k}$, in general, can satisfy the predetermined condition $\left(\left\|\boldsymbol{H}_{o, k}-\boldsymbol{H}^{(j)}\right\| \leq \delta, \delta\right.$ is a very small quantity), particularly in instances where the cable-driven parallel robot locates at the symmetric geometric position, and therefore satisfy the nonlinear equation (4).

In the context of the design of large workspace cabledriven parallel robots, besides the cable tension distributions, one of the main aspects to take into account is the cable capabilities. Actually, the cables have to be able to support the maximal cable tension all over the desired workspace. The maximal tension is also involved in the proper determination of the cable. The cables employed here can be chosen directly from the maximal tension. In fact, for most cables, the maximal supported tension has to satisfy the equation (7). When the cable model with non-negligible cable mass is used, the determination of the appropriate cable can be done by determining the maximal tension that can occur in the cable over the whole workspace.

As far as I am concerned, when cable mass is taken into account, the cable sags appear. Indeed, contrary to straight cables, the cable tensions with non-negligible masses are not constant along the profile. Kozak [7] has shown that the cable sags have effects on the positioning of the platform. But, the cable tensions are also significantly affected by the mass of the cables [1]. Actually, the actuators and the cables have to support a part of the platform mass, but also the mass (or a part of it) of the hanging cable. Thus, while taking cable mass into account, the maximal tension $T_{\text {imax }}$ will be higher than with the simple model of cable, because:

$$
T_{\text {imax }}=\sqrt{H_{i}^{2}+\left(V_{i}-\rho g L_{i}\right)^{2}}
$$

It is noted that $\boldsymbol{V}_{i}$, in the paper, is consider to be negative as shown in Fig. (1). The problem here is that the cable tensions in the cables are directly dependent on the cable masses and meanwhile have to satisfy the equation (7). Therefore, when obtaining the cable tensions with the presented iterative optimization algorithm, the maximal tension $T_{\text {imax }}$ is required to satisfy the equation (7), and furthermore, the obtained cable tensions, in the case, are rational.

\section{SIMULATION EXAMPLES}

\subsection{Description of the Cable-Driven Parallel Robot Stud- ied}

The cable-driven parallel robot considered in the study is a point mass one, that is to say, a 4 cables / 3 DOF spatial robot which is used to realize the aerial panoramic photographing [9]. In more detail, the 3 DOF are the three translations along the $x, y$ and $z$ axes. The cable-driven parallel robot considered here is called camera robot. The 4 cable exit points are located at the 4 extremities of a rectangle. And the density of the cable used here is $0.188 \mathrm{Kg} / \mathrm{m}$. The end effector mass has been chosen to be $50 \mathrm{Kg}$. The boundary conditions of the cable tensions are presented as follows: $\boldsymbol{T}_{\mathrm{s}, \max }=\left[\begin{array}{lllll}10000 & 10000 & 10000 & 10000\end{array}\right]^{\mathrm{T}} \mathrm{N}, \boldsymbol{T}_{\mathrm{s}, \min }=\left[\begin{array}{lll}10 & 10 & 10\end{array}\right.$ $10]^{\mathrm{T}} \mathrm{N}$. The dimensions of the structure have been chosen to allow us to see the effect of cable mass deliberately. Thus, the positions of the exit points of each cable, expressed in the fixed frame, are given in Table 1. The dimensions of the structure have been chosen to allow us to see the effect of cable mass deliberately.

A spatial sloping straight line is selected to depict the significant effects of the cable sags on the tensions in the cables. It should be pointed that the starting position of the trajectory is $(25,25,5)^{\mathrm{T}} \mathrm{m}$; while the ending position of the trajectory is $(65,53,18)^{\mathrm{T}} \mathrm{m}$. It takes ten seconds from the starting position to the ending position of the trajectory above.

In order to better illustrate and understand the significant effects of cable sags on the cable tensions, the relative differences denoted by $\varepsilon$ between the cable tensions with the catenary model and the ones with the massless straight line model can be used to assess the divergences of the cable tensions, and it can be expressed as follows:

$\varepsilon=\frac{T^{c}{ }_{i}-T^{s}{ }_{i}}{T^{s}{ }_{i}} \times 100 \% \quad i=1,2,3,4$

where $T_{i}^{c}$ is the tension in the cable $i$ at cable end $\boldsymbol{B}_{i}$ obtained by the catenary model and $T_{i}^{s}$ the tension in the cable $i$ obtained by the massless straight line model.

And furthermore, the relative differences of the cable tensions denoted by $\omega$ between the first node and the last node with the catenary model can be used to evaluate the divergences of the cable tensions at the two nodes of the cable catenary, and it can be expressed as follows: 
Table 1. Exit point positions.

\begin{tabular}{|c|c|c|c|}
\hline & $\boldsymbol{x}(\mathbf{m})$ & $\boldsymbol{y}(\mathbf{m})$ & $\boldsymbol{z}(\mathbf{m})$ \\
\hline \hline Position of 1\# pulley & 0 & 0 & 23 \\
\hline Position of 2\# pulley & 100 & 0 & 23 \\
\hline Position of 3\# pulley & 100 & 90 & 23 \\
\hline Position of 4\# pulley & 0 & 90 & 23 \\
\hline
\end{tabular}

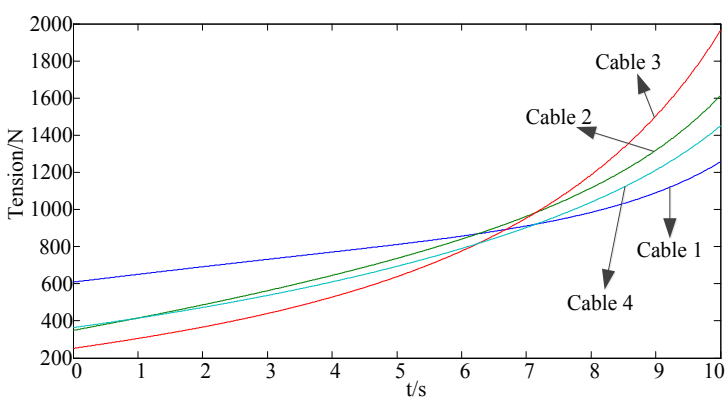

(a) Tensions with the straight line model

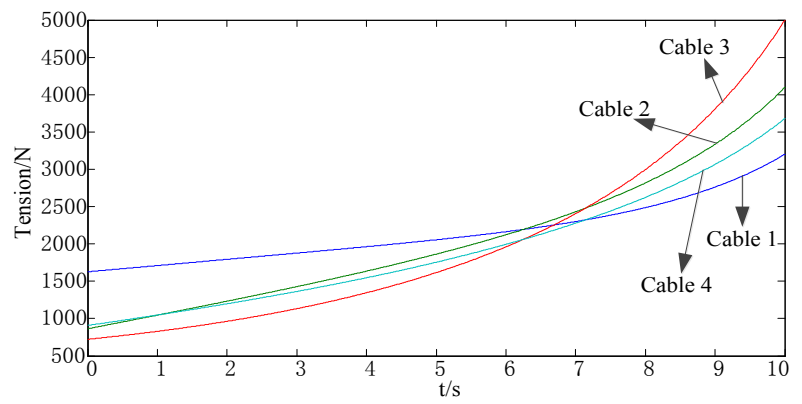

(b) Tensions with the catenary model

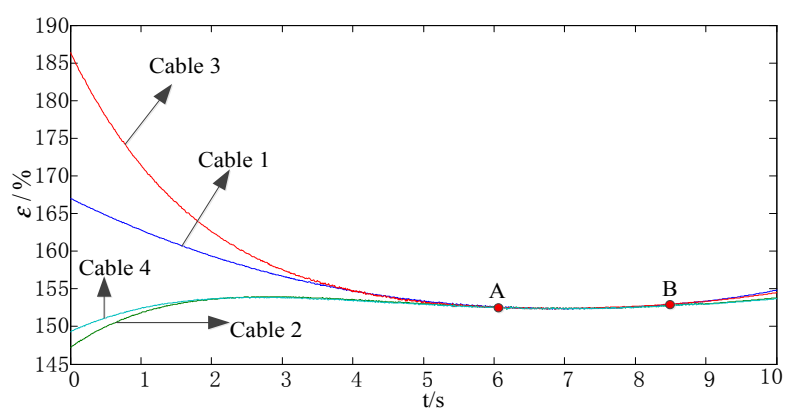

(c) Relative differences $\varepsilon$

Fig. (3). Cable tensions along the sloping straight line with the two models and their Relative differences $\varepsilon$.

$\omega=\frac{T_{\text {imax }}-T_{i}}{T_{i}} \times 100 \% \quad i=1,2,3,4$

where $T_{\text {imax }}$ is the tension in the cable $i$ at the last node (the 4 cable exit points) obtained by the catenary model and $T_{i}$ the tension in the cable $i$ at the last node $\boldsymbol{B}_{i}$ obtained by the catenary model.

\subsection{Results and Discussion}

As shown in Fig. (3), the cable tensions of the four cables along the spatial sloping straight line with the catenary and straight line models are shown to demonstrate the significant effects of cable sags on the cable tensions. As it is clearly seen in this figure, the cable tensions computed by the two models, indeed, are quite different. It should be pointed in 


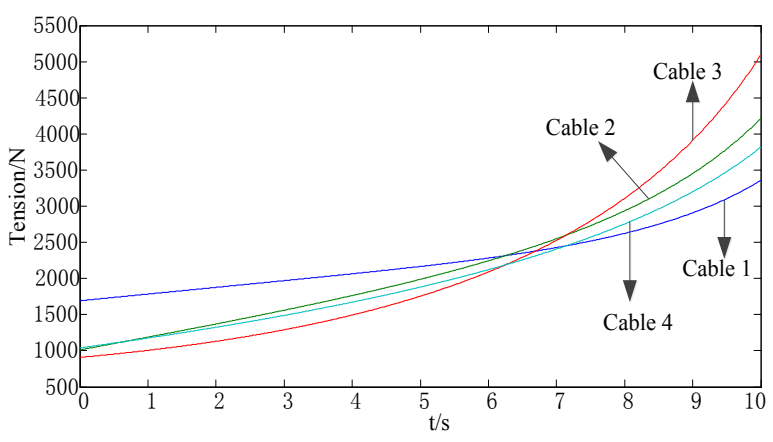

(a) Maximal tensions with the catenary model

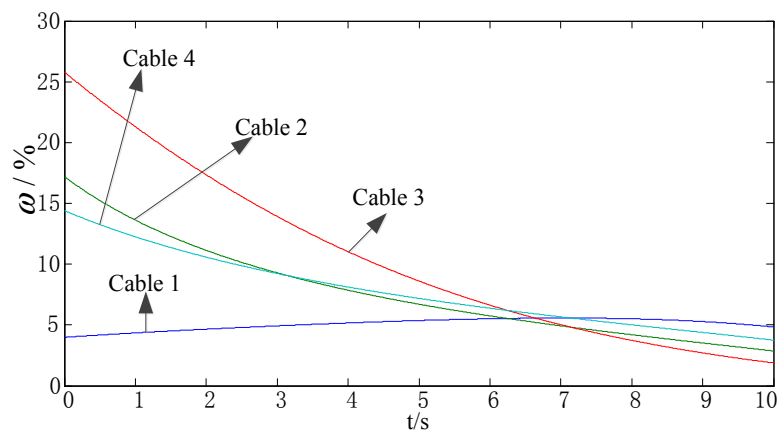

(b) Relative differences $\omega$

Fig. (4). Cable tensions along the sloping straight line with the two models.

the Fig. (3a) and (3b) that the cable tensions obtained by the catenary model are about 2 times larger than the ones by the straight line model, and this is because the non-negligible cable mass has an important effect on the cable tensions. Particular emphasis was placed on the cable tensions at the starting and the ending position, and it is perfectly clear that the cable tension at the ending position is bigger than the one at the starting position this is because the elevation at the ending position is higher than the one at the starting position. Indeed, increasing the elevation leads to an increase in the angles between the tensions $T i$ and their vertical components $V i$, so the cable tensions must increase to withstand the weights of the cables and the platform. Consequently, the tensions of the four cables at the ending point are larger than the ones at the starting point. In addition, the cable tensions, without exception, tend to increase with increasing height along $z$-direction using the two models.

Further enhancing the attraction to the relative difference, it is clearly seen from Fig. (3c) that the maximal relative difference is up to $186 \%$. It can be seen that the relative differences of the cable tensions from the point A $(x=48.96$, $y=41.772, \quad z=12.787)$ to point $\mathrm{B}(x=58.96, \quad y=48.772$, $z=16.037)$ are same in the four cables, and this is because these platform positions locate at the geometric symmetrical position of the workspace, and therefore, there is a same effect of the cable sags on the cable tensions. Note that presented Fig. (3c) the relative differences of cable tensions in cable 1 and cable 3 at the ending position are less than the one at the starting position, while are more in cable 2 and cable 4 , this is because the starting position of the sloping straight line is away from the first and third towers, and therefore, there is an obvious effect of the cable sags on the cable tensions.

Referring in Fig. (4), the maximal cable tensions and the relative differences $\omega$ along the spatial sloping straight line with the catenary model are shown to demonstrate the difference of cable tensions along the profile of the cable catenary. It can be seen that the maximal cable tensions along the profile of the cable catenary are more than the ones shown in Fig. (3b). And furthermore, it is clear from Fig. (4b) that the maximal relative difference $\omega$ is about $26 \%$. Thus, taking the maximal cable tensions of the cable catenary model into consider it is required to confirm that the cables do not exceed the restriction of the cable tensions.

From the results it can be concluded that the tension solutions obtained from the proposed algorithm are continuous and smooth. According to these results, cable tensions seem to be the parameter that is mostly affected by the nonnegligible cable mass model. It can be noticed that the mean difference between the tensions in the two models is about $155 \%$ more for the model including cable mass. Using this model turns out to be essential to an appropriate design of the robot, i.e. to the choice of the motors able to supply enough torques, but also to the dimensioning of the structure to prevent from deformations, or breakdown. 


\section{CONCLUSION}

This paper addresses a main issue related to the cabledriven parallel robots: the computation of the optimal cable tensions for a cable-driven parallel robot due to the sags in its cables. The process for solving the problem begins from the basic analysis of a single cable, which, in turn, is based on the static displacement of that cable. A cable-driven parallel robot has infinite tension solutions for a particular pose due to actuation redundancy. In this paper, a tension optimization algorithm, using an iterative optimization method, is proposed to obtain the optimal cable tensions. This research can readily be applied to other manipulators for which cable sag is significant to better analyze those manipulators. Furthermore, this research can potentially be extended to address other important issues, such as kinematics problem to account for cable sags and incorporating cable-sags predictions into the manipulator design process.

\section{CONFLICT OF INTEREST}

The authors confirm that this article content has no conflict of interest.

\section{ACKNOWLEDGEMENTS}

This work was financially supported by the National Science Foundation of China (51175397, 51105290).

\section{REFERENCES}

[1] N. Riehl, M. Gouttefarde, and S. Krut, "Effects of non-negligible cable mass on the static behavior of large workspace cable-driven parallel mechanisms", In: IEEE International Conference on Robotics and Automation, ICRA, Kobe, Japan, pp. 2193-2198, 2009.

[2] X. Liu, "On the Mechanism Performance Analysis and Motion Control of the Two Types of Parallel Manipulators", Ph.D. thesis, Xidian University, China, 2009.

[3] N. Riehl, M. Gouttefarde, and C. Baradat, "On the determination of cable characteristics for large dimension cable-driven parallel mechanisms", In: IEEE International Conference on Robotics and Automation, Alaska, USA, pp. 4709-4714, 2010.

[4] R. Verhoeven, "Analysis of the Workspace of Tendon-Based Stewart Platform", Ph.D. thesis, University of Duisburg-Essen, Duisburg, 2004

[5] P. H. Borgstrom, B. L. Jordan, and G. S. Sukhatme, "Rapid computation of optimally safe tension distributions for parallel cabledriven robots," IEEE Transactions on Robotics, vol. 25, no. 6, pp. 1271-1281, 2009.

[6] W. B. Lim, S. H. Yeo, and G. Yang, "Tension optimization for cable-driven parallel manipulators using gradient projection", In: IEEE/ASME International Conference on Advanced Intelligent Mechatronics (AIM2011), Budapest, Hungary, 2011, pp. 73-78.

[7] K. Kozak, Q. Zhou, and J. Wang, "Static analysis of cable-driven manipulators with non-negligible cable mass", IEEE Transaction on Robotics, vol. 22, no. 3, pp. 425-433, 2006.

[8] R. D. Nan, "Five-hundred-meters aperture spherical radio telescope fast," Science in China Series G: Physics, Mechanics \& Astronomy, vol. 35, no. 5, pp. 449-466, 2005.

[9] Y. Su, Y. Y. Qiu, and P. Liu, "Optimal cable tension distribution of the high-speed redundant driven camera robots considering cable sag and inertia effects", Advances in Mechanical Engineering, vol. 2014, pp. 1-11, 2014.

[10] J. L. Du, X. C. Duan, and H. Bao, "Static stiffness of a cablesupporting system with the cable sags effects considered", Journal of Mechanical Engineering, vol. 46, no. 17, pp. 29-34, 2010.

[11] J. L. Du, D. Wen, and H. Bao, "Cable vibration analysis for large workspace cable-driven parallel manipulators", In: Cable-Driven Parallel Robots, Berlin, Heidelberg, pp. 437-449, 2013.

[12] N. Riehl, M. Gouttefarde, and F. Pierrot, "On the static workspace of large dimension cable-suspended robots with non-negligible cable mass", In: ASME 2010 International Design Engineering Technical Conferences and Computers and Information in Engineering Conference, Quebec, Canada, pp. 261-270, 2010.

[13] M. Gouttefarde, J. F. Collard, and N. Riehl, "Simplified static analysis of large-dimension parallel cable-driven robots", In: IEEE International Conference on Robotics and Automation, ICRA 2012, Minnesota, USA, pp. 2299-2305, 2012.

[14] S. Fang, D. Franitza, and M. Torlo, "Motion control of a tendonbased parallel manipulator using optimal tension distribution", IEEE/ASME Transactions on Mechatronics, vol. 9, no. 3, pp. 561$568,2004$.

[15] C. Gosselin, and M. Grenier, "On the determination of the force distribution in overconstrained cable-driven parallel mechanisms", Mechanica, vol. 46, no. 1, pp. 3-15, 2011.

[16] P. Liu, Y. Y. Qiu, and Y. Su, "On the minimum cable tensions for the cable-based parallel robots", Journal of Applied Mathematics, vol. 2014, pp. 1-8, 2014 